\title{
Observations of a Post-Eruptive Arcade on October 22, 2001 with CORONAS-F, other Spaceborne Telescopes, and in Microwaves
}

\author{
V.N. Borovik ${ }^{1}$, V.V. Grechnev ${ }^{2}$, O.I. Bugaenko ${ }^{3}$, S.A. Bogachev ${ }^{3}$, \\ I.Y. Grigorieva ${ }^{1}$, S.V. Kuzin ${ }^{4}$, S.V. Lesovoi ${ }^{2}$, M.A. Livshits ${ }^{5}$, \\ A.A. Pertsov ${ }^{4}$, G.V. Rudenko ${ }^{2}$, V.A. Slemzin ${ }^{4}$, A.I. Stepanov ${ }^{5}$, \\ K. Shibasaki ${ }^{6}$, A.M. Uralov ${ }^{2}$, V.G. Zandanov ${ }^{2}$ and I.A. Zhitnik ${ }^{4}$ \\ ${ }^{1}$ Main Astronomical Observatory, St. Petersburg, Russia, email: borovik@MK4099.spb.edu \\ ${ }^{2}$ Institute of Solar-Terrestrial Physics, Irkutsk, Russia \\ ${ }^{3}$ Sternberg Astronomical Institute, Moscow, Russia \\ ${ }^{4}$ P.N.Lebedev Physical Institute, Moscow, Russia \\ ${ }^{5}$ Institute of Terrestrial Magnetism, Aeronomy and Radiowave Propagation, Troitsk, Russia \\ ${ }^{6}$ Nobeyama Radio Observatory, Japan
}

\begin{abstract}
Using multi-spectral data, we estimate plasma parameters in the post-eruptive arcade observed on October 22, 2001 at $100 \mathrm{Mm}$ above the limb: the temperature is $6 \mathrm{MK}$ and the plasma density is $(5-9) \cdot 10^{9} \mathrm{~cm}^{-3}$. We state a problem of the long-term equilibrium of the hot top of the arcade high in the corona: either the magnetic field surrounding the arcade well exceeds that one extrapolated in the potential approximation, or $\beta>1$ both inside and outside the arcade. A downflow observed in soft X-rays can contribute to the equilibrium.
\end{abstract}

Keywords. Sun: activity, coronal mass ejections, UV radiation

\section{Instrumentation, observations, and methods}

The CORONAS-F space solar observatory was launched in August 2001. The SPIRIT complex aboard the CORONAS-F contains EUV and soft X-ray telescopes and spectroheliographs providing full-disk solar images in $175 \AA$ (FeIX-XI, $T_{e} \sim 1.5 \mathrm{MK}$ ), 284, 304, and $8.42 \AA$ (MgXII, $\left.T_{e} \sim 5-15 \mathrm{MK}, T_{\max } \approx 9 \mathrm{MK}\right)$. A post-eruptive arcade on October 22, 2001 looks in MgXII channel like a 'ball' (Fig. 1) cospatial with the arcade observed with Yohkoh/SXT. The NoRH show the arcade at $17 \mathrm{GHz}$ and the SSRT at $5.7 \mathrm{GHz}$. The RATAN-600 shows a polarized source cospatial with the arcade in one-dimensional scans at several frequencies from 2.8 to $15.6 \mathrm{GHz}$. Averaged NoRH images also show two oppositely polarized regions with the arcade in between. Yohkoh/SXT images shows dark features moving down onto the arcade from above.

\section{Estimations and simulations}

Temperature of the main part of the arcade including its brightest portion is $6 \mathrm{MK}$, and its upper part has a temperature of up to $8 \mathrm{MK}$, as obtained from Yohkoh/SXT data. The plasma density within the brightest part of the arcade is $5 \cdot 10^{9} \mathrm{~cm}^{-3}$. Optically thin free-free radio emission calculated from Yohkoh/SXT data fits microwave observations both visually and quantitatively to confirm the estimates of plasma parameters. The potential magnetic field extrapolation into the corona from the SOHO/MDI magnetogram corresponds to the magnetic configuration revealed from the microwave data. Calculated 


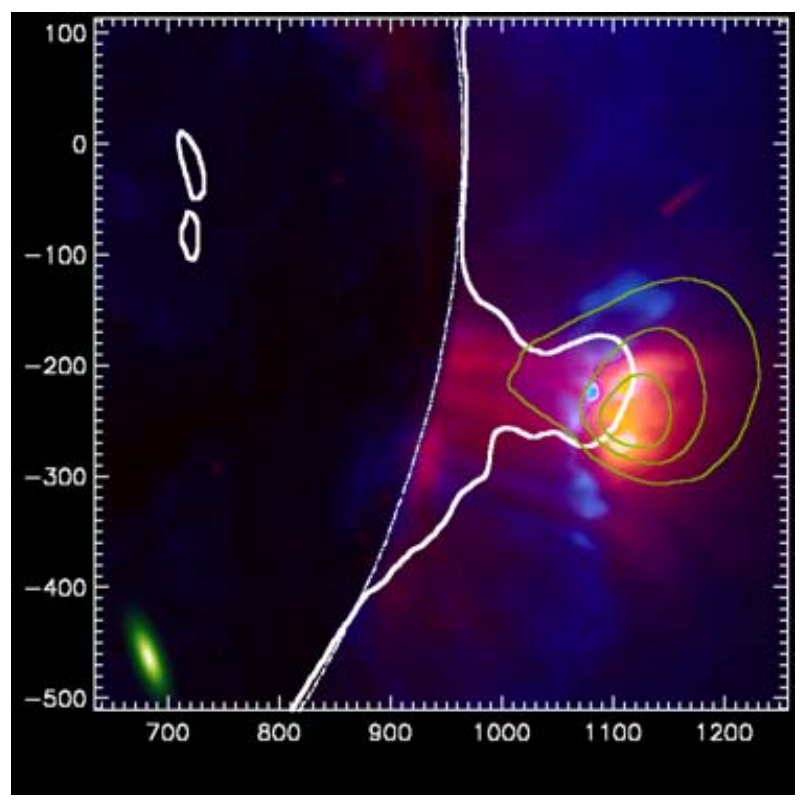

Figure 1. Arcade images. Background: EIT $195 \AA$ (blue) and SXT (red-yellow) composite. Contours: white-NoRH $\left(10^{4} \mathrm{~K}\right)$, green-SPIRIT $8.42 \AA$. Green ellipse: NoRH beam.

magnetic field is $\sim 7 \mathrm{G}$ at $\sim 100 \mathrm{Mm}$. Magnetic pressure at the arcade top turns out less than plasma pressure. The minimum required magnetic field strength of $20 \mathrm{G}$ corresponds to the equality of the gas pressure inside the arcade to the outer magnetic pressure. As for the dark features flowing into the arcade top, we have found hints that the inflow regions are slightly cooler than their vicinity. This is possible if they are falling stuff, which can contribute to mass supply to the arcade and to its equilibrium.

\section{Summary and conclusions}

(a) Plasma parameters in the post-eruptive arcade: $N_{e} \sim 10^{10} \mathrm{~cm}^{-3}, T_{e} \sim 6 \mathrm{MK}$.

(b) The large-scale magnetic configuration surrounding the arcade high in the corona revealed from high-sensitivity NoRH and RATAN-600 data is in accord with the expected one from the potential magnetic fields extrapolation into the corona.

(c) Our results give rise to a problem of the long-term equilibrium of giant hot structures high in the corona: either the magnetic field surrounding the arcade well exceeds $20 \mathrm{G} 100 \mathrm{Mm}$, or $\beta>1$ both inside and outside the hot post-eruptive arcade.

(d) Dark features moving downward onto the arcade can have influence for the problem just mentioned. They likely show the downflow due to the falling down remnants of the filament pre-ejected. This downflow can contribute to the mass supply into the arcade and to its equilibrium.

\section{Acknowledgements}

We thank the instrumental teams of the NoRH, SSRT, RATAN-600; CORONAS-F \& Yohkoh satellites for the open-data policies. This work was supported by the Russian Foundation of Basic Research under grants 02-02-16548, 03-02-16591, 03-02-17528, 0302-17357, the Ministry of Industry and Science under grants 477.2003.2, 16 KI, OFN 18; and the Presidium of RAS under grant 7/2004. 